\title{
Planckon densely piled vacuum
}

\author{
Shun-Jin Wang \\ Center for Theoretical Physics, College of Physical Science and Technology, Sichuan University, Chengdu 610064, China
}

\section{Email address:}

sjwang@home.swjtu.edu.cn

\section{To cite this article:}

Shun-Jin Wang. Planckon Densely Piled Vacuum. American Journal of Modern Physics. Special Issue: New Science Light Path on Cosmological Dark Matters. Vol. 4, No. 1-1, 2015, pp. 10-17. doi: 10.11648/j.ajmp.s.2015040101.13

\begin{abstract}
Based on the vacuum microscopic quantum structure model: the planckon densely piled vacuum model and the principle of cosmology, with the Planck era as initial conditions, we have solved the Einstein-Friedmann equations to describe the evolution of the universe. The main results are: i) the solution of Einstein-Friedmann equations have yielded the observed result: the ratio of dark energy density to vacuum quantum fluctuation energy density $\rho_{d e} / \rho_{v a c} \sim\left(t_{p} / T_{0}\right)^{2} \sim 10^{-122} s($ the Planck time $t_{p}=10^{-43} \mathrm{~s}$ and the universe age $\left.T_{0}=10^{18} \mathrm{~s}\right)$; ii) at the inflation time $t_{\text {inf }}=10^{-35} \mathrm{~s}$, the calculated universe radiation energy density is $\rho\left(t_{\text {inf }}\right) \sim 10^{-16} \rho_{\text {vac }}$ corresponding to the phase transition temperature $E_{c} \sim 10^{15} \mathrm{GeV}$ consistent with the GUT theory; iii) the expanding universe with vacuum as its environment is a non-equilibrium open system constantly exchanging energy with vacuum; during its expansion, the planckons in the universe lose quantum fluctuation energy and create the cosmic expansion quanta-cosmons, the energy of the cosmons is the lost vacuum quantum fluctuation energy and contributes to the universe energy with the calculated value $E_{\text {cosmos }}=10^{22} M_{\text {solar }} c^{2}$ (where $M_{\text {solar }}$ is solar mass) consistent with astronomic data; iv) since all planckons in the vacuum of the expanding universe lose quantum fluctuation energy resulting in hole excitations as negative gravity energy and the lost energy of planckons is used to create cosmons which in turn convert into different kinds of universe energy, the negative gravity energy plus the positive universe energy is zero; v) the induced negative gravity potential and the gravity acceleration due to the creation of cosmons are derived with the nature of radially outwards repulsive force, indicating that the cosmon may be the candidate of the dark energy quantum; vi) both the initial solution (the Planck era solution or the planckon solution) and the infinite asymptotic solution of the Einstein-Friedmann equations are unstable: the former tends to expand and the latter tends to shrink, so that the Einstein-Friedman universe undergoes a cyclic evolution of successive expansion and shrinking.
\end{abstract}

Keywords: Planckon, Expanding Universe, Dark Energy, Dark Matter

\section{Introduction}

The accelerating expansion of the universe and the dark energy are established basic facts in the exact modern cosmology $[1,2]$. To explain the dark energy as vacuum quantum fluctuation energy is a big step to reach the great goal of unifying quantum theory, relativity, and cosmology [3-8]. However, this effort has met serious challenge: the ratio of the dark energy density $\rho_{d e}$ to the vacuum quantum fluctuation energy density $\rho_{\text {vac }}$ shows a huge hierarchy difference of 122 order of magnitude [9]: $\rho_{d e} / \rho_{\text {vac }} \sim 10^{-122}$. This article is based on vacuum microscopic quantum structure model-the planckon densely piled vacuum model [10] and the principle of cosmology. With the Planck era as initial conditions, we have solved the Einstein-Friedmann equations to describe the evolution of the universe, a simple and reasonable relation between the dark energy density $\rho_{d e}$ and the vacuum quantum fluctuation energy density $\rho_{v a c}$ is obtained.

\subsection{Basic Brick of the Vacuum: The Planckon}

We have proposed a novel model of vacuum which is piled up densely by extremely tiny radiation quantum spheres with Planck radius and is a kind of a liquid crystal under mean field and semi-classical approximation. The radiation quantum sphere with the Planck radius $r_{p}$, called planckon, is made of localized standing radiation waves on average with the radius $r_{p} \sim 10^{-33} \mathrm{~cm}$, mass $m_{p} \sim 10^{-5} \mathrm{~g}$, energy $\varepsilon_{p} \sim 10^{19} \mathrm{GeV}$, and spin $s_{p}=\hbar / 2$.

There are three ways to find the parameters of the planckon with the same results. The first way requires that the planckon is a black hole with its radius $r=2 \mathrm{Gm} / \mathrm{c}^{2}$, which is made of a quantum standing wave on the $r$-sphere with wave length $\lambda=4 \pi r$, wave vector $k=2 \pi / \lambda=1 / 2 r$, quantized energy $\varepsilon(r)=\hbar k c=\hbar c / 2 r=\hbar \omega$, and mass $m(r)=$ 
$e(r) / c^{2}=\hbar / 2 r c$. From above requirements, one obtains radius, energy, mass, and spin of the planckon as follows:

$$
\begin{gathered}
r_{p}=\sqrt{\frac{\hbar G}{c^{3}}}, \varepsilon_{p}=\frac{\hbar c}{2 r_{p}} \\
m_{p}=\frac{1}{2} \sqrt{\frac{c \hbar}{G}}, s_{p}=m_{p} c r_{p}=\frac{1}{2} \hbar
\end{gathered}
$$

The planckon is the smallest microscopic quantum black hole with spin $\hbar / 2$ and made of localized radiation standing wave on the $r_{p}$-sphere with wave length $\lambda_{p}=4 \pi r_{p}$. It is also the smallest microscopic quantum particle with the heaviest mass.

Summary of the Planckon parameters: scales of space-time $r_{p}=\sqrt{\frac{\hbar G}{c^{3}}}$ and $t_{p}=\sqrt{\frac{\hbar G}{c^{5}}} ;$ volume $v_{p}=4 \pi \frac{r_{p}{ }^{3}}{3} ;$ spin $s_{p}=\frac{\hbar}{2}$; mass $m_{p}=\frac{1}{2} \sqrt{\frac{\hbar c}{G}}=\frac{\hbar}{2 c r_{p}}$; energy $\varepsilon_{p}=m_{p} c^{2}=\frac{\hbar c}{2 r_{p}}=\hbar \omega_{p}$; zero energy $\varepsilon_{p 0}=\frac{\varepsilon_{p}}{2}=\frac{\hbar c}{4 r_{p}}$; zero energy density of Planckon $\rho_{p 0}=\frac{\varepsilon_{p 0}}{v_{p}}=\frac{3}{16 \pi} \frac{c^{4}}{G^{2} \hbar}=\frac{\rho_{p}}{2}\left(\varepsilon_{p 0}=\rho_{p 0} v_{p}=\frac{\hbar c}{4 r_{p}}\right)$; the vacuum zero energy density is twice that of Planckon (since vacuum is made of densely piled planckons and each planckon has two spin states): $\rho_{v a c}=\rho_{p}=2 \rho_{p 0}=\frac{3}{8 \pi} \frac{c^{7}}{G^{2} \hbar}, \rho_{v} v_{p}=\varepsilon_{p}=$ $\frac{\hbar c}{2 r_{p}}$.

\subsection{Planckon Densely Piled Vacuum}

There are two ways (phases) to densely pile vacuum by spherical planckons [10]. Face-center cubic crystal: pile layer order is $\mathrm{ABCABCABC...,pile} \mathrm{period} \mathrm{is} \mathrm{ABC}$, and hexagon dense crystal: pile layer order is $\mathrm{ABABABABAB} \ldots$, pile period is $\mathrm{AB}$.

For infinite crystal, the two phases maybe degenerate in energy. However, for finite crystal, due to dislocation or defects, their energies become non-degenerate. For a cubic with side length $R$ (for a sphere with radius $\mathrm{R}$, the result is the same), the volume is $V=R^{3}$, the smallest dislocation volume is $\Delta V=3 R^{2} \Delta R=6 R^{2} r_{p}$ with the smallest $\Delta R=2 r_{p}$. Hence $\Delta V / V \sim 6 r_{p} / R$. For the mass density $\rho=m / V$, the ratio of the mass density change $\Delta \rho$ related to the dislocation $\Delta V$ to the mass density $\rho$ is $\Delta \rho / \rho=6 r_{p} / R$; for vacuum energy density $\rho=\rho_{p}$, dislocation energy and mass per Planckon on average is: $\Delta \rho v_{p}=M c^{2}, e_{p}=\rho_{p} v_{p}=m_{p} c^{2}, M / m_{p} \sim 6 r_{p} / R$; for proton $\left(R_{p} \sim 10^{-13} \mathrm{~cm}\right)$, dislocation mass is $M_{p} \sim 6 r_{p} m_{p} / R_{p} \sim$ $10^{-24} \mathrm{~g}$. The causes and the mechanism of dislocation of the planckon piled vacuum are closely related to the creation and formation of elementary particles with masses, it is thus a very important and basic issue for further investigation.

The solid property of vacuum comes from its extremely large mass density and stress coefficients, and its liquid property is due to the quantum fluctuation of the planckon and the related variability of the localized radiation waves. Thus the planckon densely piled vacuum is a kind of liquid crystal.

Based on the above vacuum model, the quantum statistical origin of the gravity of Schwarzschild black hole, the quantum fluctuation energy loss of vacuum in expanding universe and the origin of dark energy have been studied with reasonable results. This article is a concise report of the main results and focuses on the evolution of our universe and dark energy.

\section{Evolution of the Universe}

\subsection{Assumptions and Equations of Cosmological Model}

The basic assumptions of this article are: i) the vacuum containing and surrounding the universe as a ubiquitous environment is consisted of densely piled planckons [10]; ii) the universe was born in the planckon piled vacuum at an explosion of a planckon of the vacuum so that it should start from the Planck era, namely the energy density, radius, and time of the planckon (the basic brick of vacuum) are its initial conditions; iii) the universe obeys the cosmological principle; iv) the evolution of the universe is conducted by the Einstein-Friedmann equations with a flat curvature $k=0$ (which can be taken off) and the energy content includes radiation, cold matter, dark matter, as well as dark energy; v) the inflation occurs at the early time $\tau_{\text {inf. }}$.

The assumptions (i) and (ii) are from a study of the microscopic quantum structure of black holes, vacuum, and gravity systems [10], the assumptions (iii) and (iv) are based on the commonly accepted physical and cosmological principles $[3,7,8]$. Since the Einstein-Friedmann equations are of the nature of classical gravitation, the quantum nature of the universe evolution should be incorporated by the initial and boundary conditions, namely the assumptions (ii) and (v).

First let us introduce the space-time scales and energy density of planckon to describe the Planck era. The planckon is the smallest brick of vacuum, which consists of quantum radiation standing wave in the sphere with the radius $r_{p}$, volume $v_{p}$, period $\tau_{p}$, and energy $\varepsilon_{p}$ as follows [10]:

$$
\begin{aligned}
& r_{p}=\sqrt{\frac{\hbar G}{c^{3}}} \sim c t_{p}, v_{p}=\frac{4 \pi r_{p}{ }^{3}}{3}, t_{p}=\sqrt{\frac{\hbar G}{c^{5}}} \\
& \varepsilon_{p}=m_{p} c^{2}=\frac{\hbar c}{2 r_{p}},
\end{aligned}
$$

where $t_{p} \sim 10^{-43} \mathrm{~s}, r_{p}=c t_{p} \sim 10^{-33} \mathrm{~cm}, \varepsilon_{p} \sim 10^{19} \mathrm{GeV}$.

Based on the planckon densely piled vacuum model, the vacuum zero energy density is just the planckon zero energy density as shown above:

$$
\begin{gathered}
\rho_{v a c}=\rho_{p}=\frac{\varepsilon_{p}}{v_{p}}=\frac{K}{\left(c t_{p}\right)^{2}} \\
K=\frac{3 c^{4}}{8 \pi G}, t_{p}=\sqrt{\frac{3 c^{2}}{8 \pi G \rho_{v a c}}}
\end{gathered}
$$

According to the above assumptions, the time evolution of the isotropic and homogeneous universe obeys the Einstein-Friedmann equations [3]:

The expanding equation: 


$$
\left(\frac{\dot{\mathrm{R}}}{R}\right)^{2}=\frac{8 \pi G \rho}{3 c^{2}}=\Lambda=\lambda^{2}, \lambda=\sqrt{\frac{8 \pi G \rho}{3 c^{2}}}
$$

The accelerating equation:

$$
\frac{\dddot{\mathrm{R}}}{R}=-\frac{4 \pi G}{3 c^{2}}(\rho+3 p)
$$

In modern cosmology, the commonly accepted point of view is that the universe energy density contains the components of radiation $\rho_{r}$, cold matter $\rho_{m}$, dark matter $\rho_{d m}$, and dark energy $\rho_{d e}$. The equation of state is as follows:

$$
\begin{gathered}
p_{i}=\omega_{i} \rho_{i}, \rho=\sum_{i} \rho_{i}, p=\sum_{i} p_{i}=\sum_{i} \omega_{i} x_{i} \rho=\omega \rho \\
\omega(t)=\sum_{i} \omega_{i} x_{i}(t), \quad x_{i}(t)=\frac{\rho_{i}(t)}{\rho(t)}, \sum_{i} x_{i}(t)=1 \\
i=r, m, d m, d e \\
\omega_{m}=\omega_{d m}=0, \omega_{r}=\frac{1}{3} \\
\omega_{d e}=-1,-1 \leq \omega(t) \leq \frac{1}{3}
\end{gathered}
$$

In the following section, we shall obtain the solutions to the Einstein-Friedmann equations, which show that during the evolution of the universe, the energy density components of $\rho_{i}(t), x_{i}(t)$, and the coefficients $\omega_{i}(t)$ of the equations of state vary in their own intervals and make the total energy density $\rho(t)$ vary in time continuously. Instead, the radial scale factor $R(t)$ of the universe will have the phase change from power law functions to exponential function or the inverse. The Einstein-Friedmann equations can describe both the inflation (exponential) phase and power law phases for the radial scale factor function $R(t)$.

\subsection{Evolution of the University Energy Density}

Equation (4) and (5) lead to:

$$
\frac{d \rho}{\sqrt{\rho}(\rho+p)}=-\sqrt{\frac{24 \pi G}{c^{2}}} d t
$$

By using the equation of state $p(t)=\omega(t) \rho(t)$, one obtains the solution:

$$
\begin{gathered}
\rho\left(t-t_{0}\right)=\rho\left(t_{0}\right) /\left\{1+\left(\frac{3}{2}\right) \sqrt{\frac{8 \pi G \rho\left(t_{0}\right)}{3 c^{2}}}\left[\left(t-t_{0}\right)+\right.\right. \\
t 0 t \omega \tau d \tau] 2
\end{gathered}
$$

In viewing the interval $[-1,1 / 3]$ of $\omega(t)$ and the $\left(t-t_{0}\right)$ term in equation (10), $\rho\left(t-t_{0}\right)$ in general, is not so sensitive to $\omega(t)$ (only when $\omega(t) \rightarrow-1, \rho\left(t-t_{0}\right)$ changes from inverse quadrature functions to constant). In contrast, $R\left(t-t_{0}\right)$ is very sensitive to $\omega(t)$ and can change from power functions to exponential function. This behavior leads to the problem of consistency between $\rho(t)$ and $R(t)$ as approximations are made. The evolution details of different energy density components $\rho_{i}(t)$ controlling the evolution of $\omega(t)$, is very important to produce a physically reasonable and realistic evolution for both $\rho(t)$ and $R(t)$, and establishes the consistency between $\rho(t)$ and $R(t)$. Since the evolution of $\rho_{i}(t)$ is related to the interactions of elementary particles under astronomic and cosmic conditions, the knowledge of particle physics is needed for a detailed description.

If the initial conditions are $t_{0}=t_{p}=\sqrt{\frac{3 c^{2}}{8 \pi G \rho_{v a c}}}$ and $\rho\left(t_{p}\right)=\rho_{p}=\rho_{v a c}$, the solution is:

$$
\rho\left(t-t_{p}\right)=\rho_{v a c} /\left\{1+\left(\frac{3}{2}\right) \frac{\left(t-t_{p}\right)}{t_{p}}\left(1+\omega_{\text {mid }}(t)\right)\right\}^{2}
$$

where $\omega_{\text {mid }}(t)$ is the integration mid-value of $\omega(t)$ from $t_{p}$ to $t$ : $\int_{t_{0}}^{t} \omega(\tau) d \tau=\omega_{\text {mid }}(t)\left(t-t_{p}\right)$.

For dark energy: $\omega_{d e}(t)=-1, \rho_{d e}\left(t-t_{p}\right)=\rho_{v a c}$; for radiation: $\omega_{r}(t)=1 / 3, \rho_{r}\left(t-t_{0}\right) \rho_{v a c} /\left[1+2\left(t-t_{p}\right) / t_{p}\right]^{2}$; for cold matter: $\omega_{m}(t)=0, \rho_{r}\left(t-t_{p}\right) \rho_{v a c} /\left[1+\frac{3}{2}\left(t-t_{p}\right) / t_{p}\right]^{2} ;$ and for the global average evolution: $\omega_{\text {mid }}(t)=-1 / 3, \rho_{r}(t-$ to) $\rho v a c / 1+(t-t p) / t p 2$.

According to the exact modern cosmology, the creation and evolution of the universe roughly have three stages: i) pre-inflation, namely from explosion to inflation point, ii) inflation, iii) after inflation, different kinds of matter are created and the evolution of the universe is controlled by their energy densities. Now let us consider the details of the evolution in three steps so that the inflation can be incorporated at the early time and the phase transition temperature can be calculated. The specific evolutions of three steps are in the following:

A) From $t_{p}=10^{-43} \mathrm{~s}$ to inflation moment $t=\tau_{\text {inf }} \sim 10^{-35} \mathrm{~s}$ (radiation dominant $\omega_{\text {mid }}=1 / 3$ ). The solution is:

$$
\begin{gathered}
\rho\left(\tau_{\text {inf }}-t_{p}\right)=\rho_{\text {vac }} \\
/\left\{1+\left(\frac{3}{2 t_{p}}\right)\left[\left(\tau_{\text {inf }}-t_{p}\right)\right.\right. \\
\left.\left.+\int_{t_{p}}^{\tau_{\text {inf }}} \omega(\tau) d \tau\right]\right\}^{2}
\end{gathered}
$$

$$
\begin{array}{r}
\sim \frac{4}{9} \rho_{v a c}\left[\frac{t_{p}}{\left(\tau_{\text {inf }}-t_{p}\right)\left(1+\omega_{\text {mid }}\right)}\right]^{2} \\
=\frac{1}{4} \rho_{v a c}\left(\frac{t_{p}}{\tau_{\text {inf }}-t_{p}}\right)^{2}
\end{array}
$$

The energy density before inflation at $\tau_{\text {inf }}$ is:

$$
\frac{\rho\left(\tau_{\text {inf }}-t_{p}\right)}{\rho_{\text {vac }}}=\frac{1}{4}\left[\frac{t_{p}}{\left(\tau_{\text {inf }}-t_{p}\right)}\right]^{2} \sim 10^{-16}
$$

The radiation energy density $\rho\left(\tau_{\text {inf }}-t_{p}\right)$, the radiation quantum energy $E_{c}$ and its wavelength $\lambda_{c}$ before inflation, and the planckon energy $\varepsilon_{p}$ and its wavelength $\lambda_{p}$ have the relation: 


$$
\frac{\rho\left(\tau_{\text {inf }-t_{p}}\right)}{\rho_{v a c}}=\left(\frac{\lambda_{p}}{\lambda_{c}}\right)^{4}=\left(\frac{r_{p}}{r_{c}}\right)^{4}=\left(\frac{E_{c}}{\varepsilon_{p}}\right)^{4}
$$

From $\varepsilon_{p}=10^{19} \mathrm{GeV}$, one obtains:

$$
\frac{\rho\left(\tau_{\text {inf }}\right)}{\rho_{\text {vac }}}=\left(\frac{E_{c}}{\varepsilon_{p}}\right)^{4} \sim 10^{-16}, E_{c} \sim 10^{15} \mathrm{GeV}
$$

which is the temperature of the GUT at the time of phase transition and indicates that the first step evolution from $t_{p}$ to $\tau_{\text {inf }}$ is reasonable and yields correct results.

B) From $\left[\tau_{\text {inf }} R\left(\tau_{i n f}\right), \rho\left(\tau_{i n f}\right)\right]$ to $\left[t_{\text {inf }}=10^{-34} s, R\left(t_{i n f}\right), \rho\left(t_{\text {inf }}\right)=\right.$ $\left.\rho\left(\tau_{\text {inf }}\right)\right]$ : inflation $\left(\omega_{\text {mid }}=-1\right)$ leads to $\rho\left(t_{\text {inf }}\right)=\rho\left(\tau_{\text {inf }}\right)$.

C) From $\left[t_{\text {inf }}=10^{-34} \mathrm{~s}, R\left(t_{\text {inf }}\right), \rho\left(t_{\text {inf }}\right)=\rho\left(\tau_{\text {inf }}\right)\right]$ to the present time $\mathrm{T}_{0}=10^{18} \mathrm{~s}$, the solution is:

$$
\begin{gathered}
\rho\left(T_{0}-t_{\text {inf }}\right)=\rho\left(\tau_{\text {inf }}\right) \\
/\left\{1+\left(\frac{3}{2 \dot{\mathrm{t}}_{\text {inf }}}\right)\left[\left(T_{0}-t_{\text {inf }}\right)\right.\right. \\
\left.\left.+\int_{t_{\text {inf }}}^{T_{0}} \omega(\tau) d \tau\right]\right\}^{2} \\
\sim \frac{4}{9} \rho\left(\tau_{\text {inf }}\right)\left[\frac{\dot{\mathrm{t}}_{\text {inf }}}{\left(T_{0}-t_{\text {inf }}\right)\left(1+\omega_{\text {mid }}\right)}\right]^{2} \\
\frac{\rho\left(T_{0}-t_{\text {inf }}\right)}{\rho\left(\tau_{\text {inf }}\right)} \sim 10^{-106}, \dot{\mathrm{t}}_{\text {inf }}=\sqrt{\frac{3 c^{2}}{8 \pi G \rho\left(\tau_{\text {inf }}\right)}} \sim 10^{-35} S
\end{gathered}
$$

where $\omega_{\text {mid }}$ is the mid-value of $\omega(t)$ from $t_{\text {inf }}=10^{-34} \mathrm{~s}$ to $T_{0}=$ $10^{18} \mathrm{~s}$. In the following, we will know that the evolution of $R(t)$ requires that $\omega_{\text {mid }}=-1 / 3$. From $\rho\left(\tau_{\text {inf }}\right)=\rho\left(t_{\text {inf }}\right)$ and $\rho\left(\tau_{\text {inf }}\right) / \rho_{\text {vac }} \sim 10^{-16} \mathrm{~s}$, one gets:

$$
\begin{aligned}
\rho\left(T_{0}-t_{\text {inf }}\right)= & \rho\left(t_{\text {inf }}\right) 10^{-106}=\rho_{\text {vac }} \frac{\rho\left(t_{\text {inf }}\right)}{\rho_{\text {vac }}} 10^{-106}= \\
& \rho_{\text {vac }} 10^{-122}
\end{aligned}
$$

Now consider one step evolution from $t_{p}$ directly to $T_{0}=$ $10^{18} \mathrm{~s}$ by using the above formulae. The ratio of the universe energy density to the vacuum quantum fluctuation energy density is:

$$
\frac{\rho\left(T_{0}-t_{p}\right)}{\rho_{\text {vac }}}=\frac{4}{9}\left[\frac{\dot{\mathrm{t}}_{p}}{\left(T_{0}-t_{p}\right)\left(1+\omega_{\text {mid }}\right)}\right]^{2} \sim 10^{-122}
$$

where $\omega_{\text {mid }}$ is the mid-value between $t_{p}$ and $T_{0}$. Since one step evolution $\left(t_{p} \rightarrow T_{0}\right)$ and three step evolution $\left(t_{p} \rightarrow \tau_{\text {inf }} \rightarrow t_{\text {inf }} \rightarrow\right.$ $T_{0}$ ) yield the same result, the time duration of phase transition thus has no effect on the energy density of the universe at the present time. It should be pointed out the initial condition of the Planck era is a key point to obtain the correct results. This implies that our universe was born in the planckon piled vacuum at an explosion of a planckon in the vacuum, not from a geometric point.

Since at the present time $T_{0}$, the dark energy density $\rho_{d e}$ is $73 \%$ of the universe total energy density, so we have:

$$
\rho\left(T_{0}\right) \sim \rho_{d e}\left(T_{0}\right), \frac{\rho_{d e}}{\rho_{v a c}}=10^{-122}
$$

\subsection{Evolution of the Universe Radius}

From the expansion equation, we have the equation for $R(t)$ :

$$
\frac{d R}{R}=\sqrt{\frac{8 \pi G \rho(t)}{3 c^{2}}} d t=\lambda(t) d t
$$

Using the obtained solution of the universe energy density $\rho(t)$ and with mid-value theorem $\int_{t_{0}}^{t} \omega(\tau) d \tau=\omega_{\text {mid }}(t)(t-$ $t 0$, one obtains the solution of $R(t)$ in terms of $\omega_{\text {mid }}(t)$.

$$
\begin{gathered}
R(t)=R\left(t_{0}\right)\left[1+\frac{1}{n} \sqrt{\frac{8 \pi G \rho\left(t_{0}\right)}{3 c^{2}}\left(t-t_{0}\right)}\right]^{n} \\
n=\frac{2}{3\left(1+\omega_{\text {mid }}(t)\right)}
\end{gathered}
$$

which is very sensitive to $\omega_{\text {mid }}(t)$. As $t$ takes a fixed value $t=$ $T, \omega_{\text {mid }}(T)=\omega_{\text {mid }}$ is the mid-value in the time interval $\left[t_{0}, T\right]$.

A) As $\omega_{\text {mid }} \rightarrow-1$ (dark energy dominant) $\mathrm{n} \rightarrow \infty$,

$$
R(t)=R\left(t_{0}\right) \exp \left[\sqrt{\frac{8 \pi G \rho\left(t_{0}\right)}{3 c^{2}}}\left(t-t_{0}\right)\right]
$$

Equation (22) is the same as that obtained before.

The velocity after inflation from $\tau_{\text {inf }}=10^{-35} \mathrm{~s}$ and $t_{\text {inf }}=10^{-33} \mathrm{~s}$, one gets:

$$
\dot{\mathrm{R}}\left(t_{\text {inf }}=10^{-33} \mathrm{~s}\right) \sim 10^{49} \mathrm{~cm} / \mathrm{s}
$$

The effect of curvature $k$ term approaches zero: $\left(\rho-\rho_{c}\right) / \rho_{c}=$ $c^{2} k / H^{2} R^{2}=c^{2} k / \dot{\mathrm{R}}^{2} \sim 10^{-78} \sim 0$, so after inflation the space becomes flat. We will show later, before inflation, the effect of curvature $k$ term is very large and space is not flat.

B) As $\omega_{\text {mid }} \rightarrow 1 / 3$ (radiation dominant)

$$
R(t)=R\left(t_{0}\right)\left[1+2 \sqrt{\frac{8 \pi G \rho\left(t_{0}\right)}{3 c^{2}}}\left(t-t_{0}\right)\right]^{1 / 2}
$$

The radial velocity at $t_{p}$ and $\tau_{\text {inf }}$ :

$$
\begin{aligned}
\dot{\mathrm{R}}(t) & =\frac{r_{p}}{t_{p}}\left[1+2 \frac{\left(t-t_{p}\right)}{t_{p}}\right]^{-\frac{1}{2}} \\
& \sim 10^{10} \cdot\left[1+2 \frac{\left(t-t_{p}\right)}{t_{p}}\right]^{-\frac{1}{2}} \mathrm{~cm} / \mathrm{s}
\end{aligned}
$$

From the above equation, one obtains the velocity at $t_{p}$ : $\dot{R}=\left(t_{p} \sim t_{p}\right) \sim 10^{10} \mathrm{~cm} / \mathrm{s} \sim c$, and the velocity before inflation at $\tau_{\text {inf }}=10^{-35} \mathrm{~s}: \dot{R}\left(\tau_{\text {inf }}\right) \sim 10^{6} \mathrm{~cm} / \mathrm{s}$. The curvature effect before inflation is large: $\left(\rho-\rho_{c}\right) / \rho_{c}=c^{2} k / H^{2} R^{2}=c^{2} k / \dot{R}^{2} \sim 10^{8}>>1$, so the space is not flat.

C) As $\omega_{\text {mid }} \rightarrow 0$ (cold matter dominant)

$$
R(t)=R\left(t_{0}\right)\left[1+\frac{3}{2} \sqrt{\frac{8 \pi G \rho\left(t_{0}\right)}{3 c^{2}}}\left(t-t_{0}\right)\right]^{\frac{2}{3}}
$$


D) The global average evolution from $t_{p}$ to $T_{0}$ : in the following, we shall show that for the global average evolution, nature requires $\omega_{\text {mid }}=-1 / 3$ which leads to:

$$
\begin{aligned}
R(t) & =R\left(t_{p}\right)\left[1+\sqrt{\frac{8 \pi G \rho\left(t_{0}\right)}{3 c^{2}}}\left(t-t_{0}\right)\right] \\
& =r_{p}\left[1+\frac{\left(t-t_{p}\right)}{t_{p}}\right]
\end{aligned}
$$

The evolution is linear.

The radial scales of the universe at $\tau_{\text {inf }}=10^{-35} \mathrm{~s}$ and $t_{\text {inf }}=$ $10^{-33} \mathrm{~s}$ :

a) The radiation dominant $\left(\omega_{\text {mid }}=1 / 3\right)$ from $\left(t_{p}, r_{p}, \rho_{\text {vac }}\right)$ to $\left(\tau_{\text {inf }}=10^{-35} s, R\left(\tau_{\text {inf }}\right), \rho\left(\tau_{\text {inf }}\right)\right)$ :

$$
\begin{aligned}
R\left(\tau_{\text {inf }}\right)= & R\left(t_{p}\right)\left[1+2 \sqrt{\frac{8 \pi G \rho\left(t_{0}\right)}{3 c^{2}}}\left(\tau_{\text {inf }}-t_{0}\right)\right]^{1 / 2} \\
& =\sqrt{2} r_{p}\left[\frac{\left(\tau_{\text {inf }}-t_{p}\right)}{t_{p}}\right]^{1 / 2} \sim 10^{-29} \mathrm{~cm}
\end{aligned}
$$

b) Inflation $\left(\omega_{\text {mid }}=-1\right)$ from $\left(\tau_{\text {inf }}, R\left(\tau_{\text {inf }}\right), \rho\left(\tau_{\text {inf }}\right)\right)$ to $\left(t_{\text {inf }}=\right.$ $\left.10^{-33} \mathrm{~s}, R\left(t_{\text {inf }}\right), \rho\left(t_{\text {inf }}\right)\right)$; from $t_{0}=\tau_{\text {inf }}, t=t_{\text {inf }}=10^{-33} \mathrm{~s}, \rho\left(t_{0}\right)$ $=\rho\left(\tau_{\text {inf }}\right), \sqrt{8 \pi \rho\left(\tau_{\text {inf }}\right) / 3 c^{2}}=1 / \tau_{\text {inf }}, \quad \tau_{\text {inf }}=10^{-35} \mathrm{~s}$, and $R\left(\tau_{\text {inf }}\right)=10^{-29} \mathrm{~cm}$, one obtains:

$$
\begin{aligned}
R\left(t_{\text {inf }}\right) & =R\left(\tau_{\text {inf }}\right) \exp \left[\sqrt{\frac{8 \pi G \rho\left(\tau_{\text {inf }}\right)}{3 c^{2}}}\left(t_{\text {inf }}-\tau_{\text {inf }}\right)\right] \\
& =10^{-29} \cdot \exp \left(\frac{t_{\text {inf }}}{\tau_{\text {inf }}}\right) \sim 10^{14} \mathrm{~cm}
\end{aligned}
$$

The evolution equation for $R(t)$ is related to energy conservation as follows:

$$
\frac{d\left(\rho R^{3}\right)}{d t}=-p \frac{d\left(R^{3}\right)}{d t}
$$

Physical implication of equation (30): as $p>0$ (radiation dominant), $d\left(R^{3}\right) / d t>0$ and $d\left(\rho R^{3}\right) / d t<0$, the expanding universe loses energy to vacuum; as $p<0$ (dark energy dominant), $d\left(R^{3}\right) / d t>0$ and $d\left(\rho R^{3}\right) / d t>0$, the expanding universe acquires energy from vacuum. Furthermore, from the above equations and discussions, one can find that as $R$ approaches infinite, the solution of the Einstein-Friedmann equations is unstable with respect to the perturbation of decreasing $\mathrm{R}$ and the universe will start to shrink inwards. On the other hand, for the initial state solution, namely the Planck era solution or the planckon solution, the compression of the planckon will make its energy density and pressure larger than those of its environment, a rebound will be resulted and the afterwards expansion of the universe will start and be conducted by the Einstein-Friedman equations. In other words, the initial state solution is also unstable and tends to expand. Therefore, the Einstein-Friedmann universe with the planckon solution as its initial condition in its expansion phase and as its smallest limit in its shrinking phase will undergo a cyclic evolution of successive expansion and shrinking.

The total energy $E$ of the universe: from $p=\omega \rho, E=\rho V$, and $V=4 \pi R^{3} / 3$, one obtains:

$$
d \ln [E]=-\omega d \ln V
$$

If $\omega$ assumes the constant mid-value $\omega=\omega_{\text {mid }}=C$, then:

$$
d \ln \left[E V^{\omega_{m i d}}\right]=0, E=\mathrm{C} / V^{\omega_{m i d}}
$$

The total energy evolution with $\mathrm{R}$ is as follows: for radiation dominance $(\omega=1 / 3), E_{r}=C / R$; for cold matter dominance $\left(\omega_{\text {mid }}=0\right), E_{m}=C$; for dark energy dominance $\left(\omega_{\text {mid }}=-1\right), E_{d e}=C R^{3}$; for global average evolution $\left(\omega_{\text {mid }}=\right.$ $-1 / 3), E=C R$.

The above evolution of energy density and total energy of the universe indicate that the expanding universe with vacuum as its environment is a non-equilibrium open system constantly exchanging energy with vacuum. The universe was born in the planckon piled vacuum at an explosion of a planckon in the vacuum, the space-time scales and the energy density of the planckon constitute its initial condition. During its expansion, more planckons are involved in the universe evolution and all the involved planckons in the universe lose their quantum fluctuation energy, and the lost irregular quantum fluctuation energy of planckons converts into cosmons and transforms into different kinds of universal energy. The expanding universe acquires energy from vacuum in the form of cosmons which are created and made of the lost quantum fluctuation energy of planckons. We will show later that the negative gravity potential induced by cosmons has the nature of outward repulsive force; the planckon may thus be the candidate of the dark energy quanta. The cold matter has no net energy exchange with vacuum.

\section{4. $\rho(r)-R$ Relation}

Combining the energy conservation equation and the radial evolution equation, one obtains the $\rho-R$ relation:

$$
\rho(R)=\rho_{v a c}\left(\frac{r_{p}}{R}\right)^{3\left(1+\omega_{m i d}\right)}
$$

Different $\omega_{\text {mid }}$ lead to different power laws: for radiation, $\omega_{\text {mid }}=1 / 3, \rho=\rho_{\text {vac }}\left(r_{p} / R\right)^{4}$; for matter, $\omega_{\text {mid }}=0, \rho=\rho_{\text {vac }}\left(r_{p} / R\right)^{3}$; for dark energy, $\omega_{\text {mid }}=-1, \rho=\rho_{\text {vac }}$; for global average evolution, $\omega_{\text {mid }}=-1 / 3, \rho=\rho_{\text {vac }}\left(r_{p} / R\right)^{2}$.

From the evolution of $\rho(t)$, one can obtain more information and the value $\omega_{\text {mid }}>-1$ leads to $\rho\left(T_{0}\right) / \rho_{\text {vac }} \sim 10^{-122}$. From the $R\left(t-t_{p}\right)$ expression:

$$
\begin{gathered}
R(t)=R\left(t_{p}\right)\left[1+\frac{1}{n} \sqrt{\frac{8 \pi G \rho\left(t_{p}\right)}{3 c^{2}}\left(t-t_{0}\right)}\right]^{n} \\
n=\frac{2}{3\left(1+\omega_{\text {mid }}\right)}
\end{gathered}
$$

One can derive that if $R\left(T_{0}\right)=10^{28} \mathrm{~cm}$ and $\ddot{R}>0$ today, then there must have $\omega_{\text {mid }} \sim-1 / 3$, namely, $R\left(T_{0}\right)=10^{28} \mathrm{~cm} \rightarrow$ $\omega_{\text {mid }} \sim-1 / 3$ and $\ddot{R}>0 \rightarrow \omega_{\text {mid }}<-1 / 3$. In fact, as $\omega_{\text {mid }} \sim-1 / 3, n$ 
$\sim 1, R\left(T_{0}-t_{p}\right)=r_{p}\left[1+\left(T_{0}-t_{p}\right) / t_{p}\right]=10^{28} \mathrm{~cm}$, which yields the acceleration $\ddot{R}\left(T_{0}\right) \sim 10^{-7} \mathrm{~cm} / \mathrm{s}^{2}$ and velocity $\dot{R}\left(T_{0}\right) \sim$ $10^{10} \mathrm{~cm} / \mathrm{s} \sim c$ at the present time.

The following calculations of $R\left(T_{0}\right)$ indicate that any single component of energy density cannot yield the observed value of $R\left(T_{0}\right)$. For radiation: $\omega_{\text {mid }} \rightarrow 1 / 3, R\left(T_{0}\right) \sim 10^{-3} \mathrm{~cm}<<$ $10^{28} \mathrm{~cm}=R_{0}$, the value is too small; for matter: $\omega_{\text {mid }} \rightarrow 0$, $R\left(T_{0}\right) \sim 10^{7} \mathrm{~cm}<<10^{28} \mathrm{~cm}=R_{0}$, the value is also too small; for dark energy $\omega_{\text {mid }} \rightarrow-1, R\left(T_{0}\right)=r_{p} \exp \left(T_{0} / t_{p}\right) \sim$ $10^{-33+0.43 \times 10^{61}} \mathrm{~cm}>>10^{28} \mathrm{~cm}=R_{0}$, the value is too large.

\subsection{Cosmic Expansion Quanta and Universe Mass}

Now let us calculate the total energy and the total mass of the universe from the cosmic expansion quanta-cosmons, which are excitations of vacuum and their energies come from the lost energies of planckons during the expansion of the universe. Since the energy $e_{\text {cosmos }}$ of cosmon (cosmic expansion quantum) is the lost energy of planckon and contributes to the universe energy, the lost energy density of planckon $\Delta \rho_{p}$ at present time is just the vacuum energy density $\rho\left(T_{0}\right)=\Delta \rho_{p}$. Yet since all planckons in the vacuum of the expanding universe lose quantum fluctuation energy resulting in negative gravity energy of the vacuum within the universe and the lost energy of planckons is used to create cosmons which in turn convert into different kinds of universe energy, as the gravity energy is included, the total energy of the universe, namely the negative gravity energy plus the positive different kinds of universe energy is zero. From the Planckon volume $v_{p}$ and its lost energy density $\Delta \rho_{p}$ $=\rho\left(T_{0}\right)$, one obtains the cosmon energy $e_{\text {cosmos }}=\rho\left(T_{0}\right) v_{p}$. From $\rho\left(T_{0}\right)=\rho_{\text {vac }}\left(t_{p} / T_{0}\right)^{2}, \rho_{\text {vac }}=\rho_{p}, \varepsilon_{p}=\rho_{\text {vac }} v_{p}$, , one obtains $e_{\text {cosmos }}=\varepsilon_{p}\left(t_{p} / T_{0}\right)^{2} \sim \varepsilon_{p}\left(r_{p} / R_{0}\right)^{2} \sim 10^{-122} \varepsilon_{p} \sim 10^{-94} \mathrm{eV}$, which is extremely small. Since during the expansion of the universe, each planckon loses its energy and creates a cosmon, the number $N_{\text {cosmon }}$ of the cosmons in the universe is just the number $N_{\text {planckon }}$ of the planckons in the universe. According to the planckon densely piled vacuum model [10]:

$$
N_{\text {cosmon }}=N_{\text {planckon }}=\left(\frac{R_{0}}{r_{p}}\right)^{3} \sim 10^{183}
$$

The universe total energy and mass are:

$$
\begin{gathered}
E_{\text {cosmon }}=e_{\text {cosmon }} N_{\text {cosmon }}=\varepsilon_{p}\left(\frac{R_{0}}{r_{p}}\right)=10^{80} \mathrm{GeV} \\
m_{p}=10^{-5} \mathrm{~g}, \varepsilon_{p}=10^{19} \mathrm{GeV} \\
M_{\text {cosmon }}=m_{p}\left(\frac{R_{0}}{r_{p}}\right)=5 \times 10^{22} M_{\text {solar }} \\
\text { solar mass } M_{\text {solar }}=2 \times 10^{33} \mathrm{~g}
\end{gathered}
$$

which is consistent with the observed value of the universe mass. Thus the universe acquires energy and mass from vacuum in the form of cosmons created simultaneously with the energy loss of planckons (namely the loss of vacuum quantum fluctuation energy) during the expansion of the universe. At a word, the lost part of the vacuum quantum fluctuation energy (the negative hole excitation energy of the vacuum) manifests itself as the negative gravity potential energy of the vacuum in the universe, while the positive energy of the created cosmons convert itself into different components of the universe positive energies.

\subsection{Temperature of Background Microwave Radiation}

At the decouple time $t_{\text {decouple }}=10^{11} \mathrm{~s}$ and according to the global average evolution, one obtains:

$$
\begin{gathered}
\frac{\rho\left(t_{\text {decouple }}\right)}{\rho_{\text {vac }}}=\left(\frac{t_{p}}{t_{\text {decouple }}}\right)^{2}=10^{-108}=\left(\frac{E_{\text {decouple }}}{\varepsilon_{p}}\right)^{4} \\
E_{\text {decouple }}=10^{-8} \mathrm{GeV} \sim 10^{5} \mathrm{~K}^{0}
\end{gathered}
$$

The radius of the universe at $t_{\text {decouple }}=10^{11} \mathrm{~s}$ is:

$$
R\left(t_{\text {decouple }}\right)=r_{p}\left(\frac{t_{\text {decouple }}}{t_{p}}\right) \sim 10^{22} \mathrm{~cm}=R_{0} 10^{-6}
$$

Due to the expansion stretching of the wavelength of the photon, the photon energy today is:

$$
E_{0}=\frac{R\left(t_{\text {decouple }}\right)}{R_{0}} E\left(t_{\text {decouple }}\right) \sim 10^{-1} K^{0}
$$

which is near the observed astronomic value $2.7 K^{0}$ (the error maybe due to the uncertainty of the decouple time or the expansion power laws for $\rho(t)$ and $R(t))$.

\section{Two Kinds of Vacuum Excitations}

Now we discuss two different kinds of energy losses of planckons and their different gravity effects. According to the planckon densely piled vacuum model [10], the black hole gravity is generated by the Casimir effect due to the cutoff of the black hole horizon surface. Suppose the universe, like a black hole, had a $R_{0}$-spherical horizon surface which provides a cutoff for radial wave modes of the planckon piled vacuum. Inside the universe, every planckon will lose energy from radial modes and the vacuum has less quantum fluctuation energy density than that in flat space and the effective quantum fluctuation temperature of the vacuum will decrease, which is the microscopic origin of gravity (this is called equilibrium space Casimir effect). The planckon inside the universe black hole has the energy loss from $\varepsilon_{p}$,

$$
\Delta \varepsilon_{p}=e_{H}=\varepsilon_{p}\left(\frac{r_{p}}{R_{0}}\right)=\frac{\hbar c}{2 R_{0}}
$$

The energy loss $\Delta \varepsilon_{p}$ of the planckon will induce negative gravity which will drive particles to the horizon. The corresponding excitation quanta of vacuum will be accumulated in horizon in the form of radiation quantum with the energy $\mathrm{e}_{H}$ and the number of the radiation quanta in the horizon is just the number $N_{H}=4\left(R_{0} / r_{p}\right)^{2}$ of the planckons in the $R_{0}$-spherical planckon layer. The total mass $M_{m}$ is the excitation quantum mass $e_{H} / c^{2}$ times the number $N_{H}=$ $4\left(R_{0} / r_{p}\right)^{2}$,

$$
M_{m}=4\left(\frac{e_{H}}{c^{2}}\right)\left(\frac{R_{0}}{r_{p}}\right)^{2}=4 m_{p}\left(\frac{R_{0}}{r_{p}}\right) \sim M_{\text {cosmos }}
$$


which is in the same order of magnitude as the universe mass $M_{\text {cosmos. }}$.

On the other hand, the expansion of the universe to the radius $R_{0}$ leads to the quantum fluctuation energy loss of the planckons in the universe in quite a different way which can be called as "non-equilibrium time Casimir effect". Since in the expanding universe, the lost energy of each planckon with volume $v_{p}$ is used to create a cosmon which contributes to the universe with the energy density $\rho\left(T_{0}\right)$, the cosmon energy should be:

$$
\begin{aligned}
e_{\text {cosmon }}=\rho\left(T_{0}\right) v_{p} & =\varepsilon_{p}\left(\frac{r_{p}}{R_{0}}\right)^{2}=\frac{\hbar c}{2 r_{p}}\left(\frac{r_{p}}{R_{0}}\right)^{2} \\
& =\frac{\hbar c}{2 R_{K}}, R_{\kappa}=R_{0}\left(\frac{R_{0}}{r_{p}}\right)
\end{aligned}
$$

They contribute the universe a total mass $M_{\text {cosmos }}$ as calculated before with $M_{\text {cosmos }} \sim 10^{22} M_{\text {solar }}$.

Now let us investigate the gravity effect due to the creation of cosmons with mean energy $e_{\cos m o n}=\hbar c / 2 R_{\kappa}=\hbar \kappa / 2 c$ and $\kappa$ $=c^{2} / R_{\kappa}$. The cosmon is a standing quantum radiation wave with the wavelength $\lambda_{\text {cosmon }}=4 \pi R_{\kappa}$ and spin $1 / 2$ [10]. From the period condition of temperature Green's function for fermions, we have $e_{\cos m o s} / k_{B} T_{\cos m o s}=\pi$, and the Hawking-Unruh formulae naturally follows $\pi k_{B} T_{\text {cosmos }}=$ $e_{\text {cosmon }}=\hbar \kappa / 2 c=\hbar c / 2 R_{\kappa}$ [10]. The gravity acceleration $\kappa$ and the negative gravity potential $\phi(R)$ in the vacuum of the universe due to the quantum fluctuation energy loss and the creation of the cosmon are related as follows: $\kappa=-d \varphi(R) / d R$ $=c^{2} / R_{\kappa}$, which leads to the negative Einstein gravity potential $\varphi(R)=-c^{2} R / R_{\kappa}$. Since the above gravity acceleration $\kappa$ is in the radial direction and outward, the gravity effect induced by the cosmons has the nature of repulsive force. Thus the cosmon as a radiation quantum of fermion-type maybe a candidate of the dark energy quantum. It is dual to the radiation quantum for the Schwarzschild black hole [10] as follows: for the cosmon of expanding universe: $e_{\cos m o n}=$ $\hbar c / 2 R_{\kappa},\left(R_{\kappa}=R_{0}\left(R_{0} / r_{p}\right)\right)$; for the radiation quantum of universe black hole: $e_{H}=\hbar c / 2 R_{0}\left(R_{0}\right.$ - universe black hole radius).

It should be noted that both the universe surface cutoff and the universe expansion lead to quantum fluctuation energy loss of all the planckons which are basic bricks of vacuum, but the mechanism is quite different. The universe surface cutoff leads to the quantum fluctuation energy loss of every planckon with the energy decrease of $\Delta \varepsilon_{P}$ (space) $=e_{H}=$ $\varepsilon_{P}\left(r_{P} / R_{0}\right)$, which is a violin cord effect (equilibrium space Casimir effect); while the universe expansion leads to the quantum fluctuation energy loss of every planckon with the energy decrease of $\Delta \varepsilon_{P}($ time $)=e_{\text {cosmon }}=\varepsilon_{P}\left(r_{P} / R_{0}\right)^{2}=e_{H}\left(r_{P} / R_{0}\right)$, which is $r_{P} / R_{0}$ time smaller than $\Delta \varepsilon_{P}($ space $)=e_{H}$ and shows the effect of outward radiation with holographic nature (non-equilibrium time Casimir effect). It can be calculated that as standing quantum waves of the cosmons in the universe, their velocity $v_{\text {cosmon }} \sim \kappa \times T_{0} \sim 10^{-51} \mathrm{~cm} / \mathrm{s}$ and acceleration $a_{\text {cosmon }}=\kappa=c^{2} r_{P} / R_{0}{ }^{2} \sim 10^{-69} \mathrm{~cm} / \mathrm{s}^{2}$ are extremely small and negligible, so that the cosmons distribute their quantum energy $e_{\text {cosmon }}$ in the universe uniformly and statically. Instead, for the radiation excitations of the universe black hole, their velocity $v_{H} \sim \kappa_{H} \times T_{0} \sim 10^{10} \mathrm{~cm} / \mathrm{s}$ and acceleration $a_{H}=\kappa_{H}=c^{2} / R_{0} \sim 10^{-8} \mathrm{~cm} / \mathrm{s}^{2}$ are sizable and not negligible, so that the radiation quanta move their energy $e_{H}$ to the universe surface. Both the expansion cosmons and the radiation quanta are excitations of vacuum which is densely piled by planckons.

From the above results and discussions, we come to the very important conclusion that within a spherical region, vacuum quantum fluctuation energy loss necessarily leads to the negative gravity potential and its acceleration is of the nature of a radially outward force: inside the supposed Universe Black Hole, vacuum quantum fluctuation energy loss due to the Casimir effect leads the gravity potential $\phi(R)$ $=-\kappa_{H} R$ and the gravity acceleration $\kappa_{H}=c^{2} / R_{0}$ is radially outward to the universe horizon, every particle will feel a radial repulsive force outwards; while inside the expanding universe, the vacuum quantum fluctuation energy loss due to the expansion of the universe will definitely induce the gravity potential $\phi(R)=-\kappa R$ and the gravity acceleration $\kappa=$ $c^{2} / R_{\kappa}=r_{p} c^{2} / R_{0}^{2}$ is also radially outward, every particle will be pushed outwards to the expanding universe horizon. In the two cases the gravity induced by the vacuum quantum fluctuation energy loss plays a similar role of repulse forces due to the radially outward gravity acceleration.

\section{Holographic Nature of Expanding Universe}

The present study tells us that our universe was born from an explosion of a planckon in the planckon densely piled vacuum. The initial condition is the Planck era consisting of the planckon space-time scales $\left(r_{p}, t_{p}\right)$ and its energy density $\rho_{\text {vac }}$. The evolution of the universe is controlled by the Einstein-Fridmann equations with different energy density components. During the evolution, more and more Planckons are involved, lose their quantum fluctuation energy, and create cosmons which change the lost irregular Planckon energies into their regular energies. The gravity effect due to the creation of cosmon quanta is to produce a repulsive gravity force outward in radial direction, and the cosmon may thus be the candidate of the dark energy quantum.

An intuitively physical picture can be presented for the time evolution of the universe energy density and the creation of cosmons as follows. In the planckon densely piled vacuum, both the universe and the vacuum constitute a compound system, each of them is an open subsystem. Planckons in vacuum as radiation sources can radiate and absorb radiation quantum energy. In the stationary universe, the radiation and absorption processes are in equilibrium, there is no net effect can be observed physically in the universe subsystem. As the universe subsystem undergoes expansion, the stationary equilibrium of the two subsystems is broken, every planckon in the expanding universe is now in non-equilibrium and radiates energy quantum as a cosmon. Therefore a net effect of cosmons in the universe subsystem can be observed physically as outwards radial radiation and the planckon piled vacuum with less radial quantum fluctuation energy induces a negative gravity potential with the radially outward strength just like a repulsive force.

Let the pressure of the planckon at its $r_{p}$-spherical surface 
be $p\left(r_{p}\right)$. As the universe has expanded to $R_{0}$-sphere, the radiation pressure of the planckon at $R_{0}$-sphere is $p\left(R_{0}\right)$. Since the pressure on a spherical surface is proportional to the energy quanta number per unit area and per unit time, the radiation energy flux, according to energy conservation, $p\left(R_{0}\right)$ is inversely proportional to the spherical surface area $\left(4 \pi R_{0}^{2}\right)$.

$$
\frac{p\left(R_{0}\right)}{p\left(r_{p}\right)} \sim\left(\frac{r_{p}}{R_{0}}\right)^{2}, p\left(R_{0}\right)=\sim\left(\frac{r_{p}}{R_{0}}\right)^{2} p\left(r_{p}\right)
$$

Using the energy density and pressure relation $p\left(r_{p}\right) \sim$ $\rho_{\text {vac }}\left(r_{p}\right)$ and $p\left(R_{0}\right) \sim \rho\left(R_{0}\right)$, finally we have:

$$
\rho\left(R_{0}\right) \sim \rho_{v a c}\left(\frac{r_{p}}{R_{0}}\right)^{2} \sim \rho_{v a c}\left(\frac{t_{p}}{T_{0}}\right)^{2}
$$

This is exactly the results obtained by directly solving the Einstein-Friedmann equations and indicates that the evolution of the universe energy density has the holographic nature of radiation propagation $[11,12]$.

The above picture implies that: the evolution of the universe energy density, the creation of cosmons and dark energy, are problems of a non-equilibrium and open system, and the non-equilibrium process is more important and essential. Only in its non-equilibrium expansion, the universe can exchange energy with vacuum, the planckons in the universe can lose energy and change its lost quantum fluctuation energy into cosmon's regular energy, finally the dark energy quanta-cosmons are created. Therefore, the dark energy is not the vacuum quantum fluctuation energy itself, it is the lost energy of planckons (namely the lost part of vacuum quantum fluctuation energy) under the condition of the universe non- equilibrium expansion.

\section{Solutions to Big Bang Model}

Solutions to the three puzzles of the Big Bang model $[7,8]$ are as follows:

A) The initial singular problem is avoided by the initial conditions of the Planck era.

B) The problem of flatness of space is solved by the following calculations: before inflation, $\dot{R}=10^{6} \mathrm{~cm} / \mathrm{s}$, $\left|\rho-\rho_{c}\right| \rho \sim c^{2}|k| / H^{2} R^{2}=c^{2}|k| / \dot{R}^{2} \sim 10^{8}>>1$, the curvature $k$ term is very large and space is not flat; after inflation, $\dot{R}=$ $10^{49} \mathrm{~cm} / \mathrm{s},\left|\rho-\rho_{c}\right| / \rho \sim c^{2}|k| / H^{2} R^{2}=c^{2}|k| / \dot{R}^{2} \sim 10^{-78} \sim 0$, the curvature $k$ term is negligible and the space becomes flat.

C) The horizon problem is solved by the following calculated results: before inflation the expansion velocity $\dot{R}\left(\tau_{\text {inf }}\right) \sim 10^{6} \mathrm{~cm} / \mathrm{s}<<c$, the causal connection of the whole universe can be established; after inflation the average radial velocity $\dot{\mathrm{R}} \sim c$, the average horizon distance $D$ is approximately equal to the average expansion distance $R$ so that $D / R \sim 1$.

\section{Acknowledgements}

This work was supported in part by the National Natural Science Foundation of China under the grant No. 10974137 and 10775100, the Doctoral Education Fund of the Education Ministry of China, and by the Research Fund of the Nuclear Theory Center of HIRFL of China

\section{References}

[1] A. G. Riess, et al, Supernova Search Team Collaboration, Observational evidence from supernovae for an accelerating universe and a cosmological constant, vol. 116. Astronomical Journal, 1998, pp.1009

[2] S. Perlmutter, et al, Discovery of a supernova explosion at half the age of the universe, vol. 51 Nature, 1998, p.51; Measurements of $\Omega$ and $\Lambda$ from 42 high-redshift supernovae, vol. 517, Astrophysical Journal, 1999, p.565; Discovery of a supernova explosion at half the age of the universe and its cosmological implications, astro-ph/9712212.

[3] S. Dodelson, Modern Cosmology, Academic Press, 2003.

[4] Q. L. Lu, Z. L. Chou, and H. Y. Guo, The kinematic effect in the classical domains and the red shift phenomena of extragalactic objects, vol. 23, Acta Physica (China), 1974, p. 225.

[5] H. Y. Guo, C. G. Huang, Z. Xu, and B. Zhou, On Beltrami model of de sitter space-time, vol. A19, Modern Physics Letters, 2004, p.1701.

[6] H. Y. Guo, C. G. Huang, Z. Xu, and B. Zhou, On special relativity with cosmological constant, vol. 331, Physics Letters A, 2004, p.1.

[7] A. Guth, Inflationary universe: A possible solution to the horizon and flatness problems, vol. 23, Physical Reviews D, 1981, p. 347.

[8] A. Lindle, A new inflationary universe scenario: A possible solution of the horizon, flatness, homogeneity, isotropy and primordial monopole problems, vol.108, Physics Letters B, 1982, p.389.

[9] S. Weinberg, The cosmological constant problem, vol. 61, Reviews of Modern Physics, 1989, p.1.

[10] S. J. Wang, i) Microscopic quantum structure of black hole and vacuum versus quantum statistical origin of gravity, arXiv:1212.5862v4[gr-qu], 2014; ii) Vacuum quantum fluctuation energy in expanding universe and dark energy, arXiv:1301.1291v4[physics.gen-ph], 2014.

[11] A. Cohen, D. Kaplan, and A. Nelson, Effective Field Theory, Black Holes, and the Cosmological Constant, vol. 82, Physical Review Letters, 1999, p. 4971.

[12] M. Li, A model of holographic dark energy, vol. 603, Physics Letters B, 2004, pp.1-5. 